\title{
Spatial and temporal characteristics of carbon emissions from livestock farming in Yunnan province by state and city
}

\author{
Rui Chen ${ }^{1}$, Ji Chen ${ }^{1, *}$ \\ ${ }^{1}$ College of Economic and Management, Yunnan Agricultural University, Kunming, China
}

\begin{abstract}
The livestock industry is a pillar industry of the rural economy and an important industry of the national economy in Yunnan Province, so it is important to study the spatial and temporal characteristics of carbon emissions from the livestock industry in Yunnan Province for the development of a modern, lowcarbon and recycling livestock industry. This study draws on provincial greenhouse gas emission factors to calculate the carbon dioxide equivalents generated by enteric fermentation and manure management of cattle, sheep, pigs and poultry in each state and city of Yunnan Province. The results show that: (1) the total carbon emissions from the livestock sector in Yunnan Province decreased from 25,643,900 t in 2008 to 24,758,200 t in 2018, with an average annual carbon emission of 30,534,500 $\mathrm{t}$ and an average annual growth rate of The average annual growth rate was $0.35 \%$, showing a characteristic of "rising first - then falling". (2) In terms of spatial and temporal evolution, the layout of the low and high livestock carbon emission areas in Yunnan is stable, while the medium and high livestock carbon emission areas fluctuate frequently and the spatial and temporal differences in carbon emissions are obvious. Finally, based on the conclusions, targeted countermeasure suggestions are put forward.
\end{abstract}

\section{Introduction}

The Food and Agriculture Organization of the United Nations (FAO), in its 2006 report entitled Livestock in the Long-Term Shadow: Environmental Issues and Options, noted that livestock production is a major contributor to the global greenhouse effect, accounting for around 18\% of global anthropogenic greenhouse gas emissions, although this varies considerably around the world [1]. The IPCC (United Nations Intergovernmental Panel on Climate Change) has pointed out in all its assessment reports that the greenhouse gas emissions caused by the rapid development of animal husbandry cannot be ignored. With China's rapid economic development and increasing population, the consumption of meat, dairy products and poultry and eggs is also increasing, making China's livestock industry a pillar industry of agriculture. On this basis, the low-carbon development of the livestock industry has become one of the important elements of agricultural economic research in the new era, and domestic scholars have conducted a lot of research on the carbon emissions of the livestock industry. Yao Chengsheng et al. used the whole life cycle evaluation method to calculate that the total carbon emissions of China's livestock industry increased from 12,669,899,000 $\mathrm{t}$ to $13,189,955,000 \mathrm{t}$, with an average annual growth rate of $0.288 \%{ }^{[2]}$. Chen Yao found that the eastern region was larger than the western region than the central region in the measurement of GHG emission efficiency of China's livestock industry, which was not consistent with the regional economic development level ${ }^{[3]}$. Xie Ting et al. used the IPCC greenhouse gas inventory calculation method to calculate the greenhouse gas emissions of livestock in central China, and used a non-linear time series model to predict that the total greenhouse gas emissions from livestock in central China in 2030 would be about 4990.06 5932.74 million $\mathrm{t}^{[4]}$. Using the LMDI model, Chen $\mathrm{Su}$ et al. concluded that among the influencing factors of livestock GHG emissions, the cumulative contribution of the economic effect to promote the effect is increasing exponentially, and the labor effect and structural effect to inhibit the effect is also increasing [5].

Yunnan's livestock industry has become a pillar industry of the agricultural economy and an important source of the national economy, becoming one of the main livestock-producing provinces in China, and is in the process of transforming from a traditional crude livestock industry to a modern, large-scale and intensive one. At the same time, Yunnan Province relies on its rich tourism resources to vigorously develop the tertiary industry, mainly tourism. How to reduce the environmental pollution caused by livestock breeding has become an issue that must be considered in the process of livestock development. In view of this, this study uses data from the Yunnan Provincial Statistical Yearbook, the IPCC 2006 Greenhouse Gas Emissions Inventory Guidelines and the Provincial Greenhouse Gas Emissions Inventory Guidelines to measure the carbon emissions from livestock farming in Yunnan's states and cities from 2008 to 2018 , to understand the spatial and temporal evolution

$\overline{{ }^{*} \text { Corresponding author: j.chn@ynau.edu.cn }}$ 
characteristics of Yunnan's livestock farming carbon emissions and to make targeted recommendations.

\section{Research methodology and data sources}

\subsection{Measuring carbon emissions from livestock}

According to research on carbon emissions by scholars at home and abroad, the main sources of carbon in the livestock industry are $\mathrm{CH} 4$ gas from intestinal fermentation of ruminants and $\mathrm{CH} 4$ and $\mathrm{H} 2 \mathrm{O}$ gas from livestock and poultry manure management. Drawing on the IPCC 2006 Guidelines for Greenhouse Gas Emission Inventories, the Provincial Guidelines for Greenhouse Gas Emission Inventories and existing research, the annual carbon emissions of each state and municipality in Yunnan province are obtained by multiplying the average annual feeding capacity of different livestock species by the greenhouse gas emission factors and summing them up. $\mathrm{CH} 4$ and $\mathrm{H} 2 \mathrm{O}$ emission factors were obtained based on the scale of livestock breeding in Yunnan Province and the Provincial Greenhouse Gas Emission Inventory Guidelines.

\subsection{Average annual stocking of livestock and poultry}

Different types of statistical yearbooks in China for livestock breeding mainly count year-end stocking and slaughter. Since livestock and poultry species have different breeding cycles, the year- end stocking of a certain livestock and poultry cannot simply be defined directly as the average annual stocking, but the average annual stocking should be adjusted according to the breeding cycle, year-end stocking and annual slaughter. According to the availability of data, this paper adopts the calculation method proposed by Yao Chengsheng et al [2]. The details are as follows: when the livestock feeding cycle is greater than or equal to 1 year, the average annual feeding quantity is directly equal to the end-of-year stocking quantity of the species. When the feeding cycle of livestock is less than 1 year, the average annual feeding quantity is equal to its feeding cycle divided by 365 and then multiplied by the annual slaughter volume of livestock. In China, the feeding cycle of pigs and poultry is 200 days and 55 days respectively, which are less than 1 year, while the feeding cycle of cattle and sheep is greater than 1 year [3]. The specific formula is as follows.

$$
Y_{i}=\left\{\begin{array}{cl}
\beta_{i}, & \text { Days } \leq 365 \\
\alpha_{i} \times \frac{\text { Days }}{365}, & \text { Days }>365
\end{array}\right.
$$

Where $\mathrm{Yi}$ is the average annual stocking of livestock and poultry in year $\mathrm{i}$ (million head), and $\beta i$ is the year-end stock of livestock in year i (10,000 head), and $\alpha i$ is the livestock output in year i $(10,000$ head), and Days is the livestock feeding cycle (days).

\subsection{Enteric fermentation emissions}

Ruminant animals such as cattle, sheep, horses and donkeys emit large amounts of $\mathrm{CH} 4$ from enteric fermentation, making them an important source of carbon emissions from livestock farming, and because China is the largest pig breeding country in the world, $\mathrm{CH} 4$ emissions from enteric fermentation of pigs are also included in the total. In this paper, the total enteric fermentation $\mathrm{CH} 4$ emissions are obtained by multiplying the average annual feeding capacity of different livestock species by the corresponding enteric fermentation $\mathrm{CH} 4$ coefficients and then summing them, with the following equation.

$$
C_{1}=\sum Y_{i} \times E F_{i 1}
$$

In equation (2): $\mathrm{C} 1$ total $\mathrm{CH} 4$ emissions from enteric fermentation of livestock and poultry (million $\mathrm{t}$ ), and $Y i$ is the average annual feeding capacity of $i$ livestock species, and $E F i 1$ is the $\mathrm{CH} 4$ emission factor for different farming scales of i livestock species (as in Table 1). According to the study by Zhao Xianghua et al. it was found that in 2014, the proportion of large-scale livestock and poultry farming in Yunnan Province was 31.12\%, 25.75\%, 15.19\% and $30.36 \%$ for pigs, dairy cattle, beef cattle and sheep respectively. Therefore, in this paper, the $\mathrm{CH} 4$ emission factors under the category of farmers' free-range farming were chosen. Due to the availability of state and city data, the average values of dairy cattle, non-dairy cattle and buffalo were used for the emission factors of cattle. For sheep, the average of the emission factors for sheep and goats was used.

Table1. Emission factors for $\mathrm{CH} 4$ from animal enteric fermentation $(\mathrm{kg} / \mathrm{head} / \mathrm{year})$

\begin{tabular}{|c|c|c|c|c|c|c|c|}
\hline $\begin{array}{c}\text { Scale of } \\
\text { farming }\end{array}$ & $\begin{array}{c}\text { Dairy } \\
\text { cattle }\end{array}$ & Non-cow & $\begin{array}{c}\text { Water } \\
\text { buffalo }\end{array}$ & Sheep & Goats & Pigs & Poultry \\
\hline $\begin{array}{c}\text { Large-scale } \\
\text { rearing }\end{array}$ & 88.1 & 52.9 & 70.5 & 8.2 & 8.9 & 1 \\
\hline $\begin{array}{c}\text { Free-range } \\
\text { farming }\end{array}$ & 89.3 & 67.9 & 87.7 & 8.7 & 9.4 & - \\
\hline Grazing rearing & 99.3 & 85.3 & - & 7.5 & 6.7 & & \\
\hline
\end{tabular}

Data source: Guidelines for the Préparation of Provincial Guebenhouse Gas Inventories

\subsection{Livestock manure management emissions}

CH4 emissions are generated during the storage and handling of livestock manure before it is reused for fertilization into the soil. Treatment methods and climatic conditions then create differences in $\mathrm{CH} 4$ emission factors for manure management in different regions of the country. The average annual stocking of different livestock species is multiplied by the corresponding $\mathrm{CH} 4$ 
emission factor and then summed to the annual livestock manure management $\mathrm{CH} 4$ emissions, as follows:

$$
C_{2}=\sum Y_{i} \times E F_{i 2}
$$

in equation (3): $\mathrm{C} 2$ total $\mathrm{CH} 4$ emissions from livestock manure management (million $\mathrm{t}$ ), and $Y i$ is the average annual feeding capacity of livestock of $\mathrm{i}$ species, and EFi2 is the manure management $\mathrm{CH} 4$ emission factor for different regions of i livestock species (see Table 2). As the list of livestock enterprises in Yunnan Province includes non-cow and buffalo breeds, the emission factors for cattle are still chosen as the average of cows, non-cows and buffaloes. The emission factors for sheep are still taken as the average of the emission factors for sheep and goats.

Table2. Emission factors for $\mathrm{CH} 4$ from animal enteric fermentation $(\mathrm{kg} / \mathrm{head} / \mathrm{year})$

\begin{tabular}{ccccccccc}
\hline Region & Type & $\begin{array}{c}\text { Dairy } \\
\text { cattle }\end{array}$ & $\begin{array}{c}\text { Non- } \\
\text { cow }\end{array}$ & $\begin{array}{c}\text { Water } \\
\text { buffalo }\end{array}$ & Sheep & Goats & Pigs & Poultry \\
\hline $\begin{array}{c}\text { South- } \\
\text { West }\end{array}$ & $\mathrm{CH}_{4}$ & 6.51 & 3.21 & 1.53 & 0.48 & 0.53 & 4.18 & 0.02 \\
& $\mathrm{H}_{2} \mathrm{O}$ & 1.88 & 0.69 & 1.197 & 0.064 & 0.064 & 0.159 & 0.007 \\
\hline
\end{tabular}

Livestock manure also generates $\mathrm{H} 2 \mathrm{O}$ emissions during processing and storage, and the emission factors can vary greatly depending on the type of livestock and the region. The total annual emissions of $\mathrm{H} 2 \mathrm{O}$ from manure management in the state and city are obtained by multiplying the average annual feeding capacity of different livestock species by the corresponding $\mathrm{H} 2 \mathrm{O}$ emission factors and summing them up as follows:

$$
C_{3}=\sum Y_{i} \times E F_{i 3}
$$

In equation (4): $\mathrm{C} 3$ total $\mathrm{H} 2 \mathrm{O}$ emissions from livestock manure management (million $\mathrm{t}$ ), and $Y i$ is the average annual feeding capacity of livestock of $i$ species, and $E F i 3$ is the manure management $\mathrm{H} 2 \mathrm{O}$ emission factor for different areas of i species of livestock (see Table 2). Emission factors for cattle and sheep are treated as above.

\subsection{Total carbon emissions}

In order to study the spatial and temporal characteristics of total $\mathrm{CO} 2$ emissions and the level of greenhouse gas emissions in Yunnan province, $\mathrm{CH} 4$ and $\mathrm{H} 2 \mathrm{O}$ emissions need to be converted into $\mathrm{CO} 2$ equivalent. According to the warming effect, 1t $\mathrm{CH} 4$ and $\mathrm{H} 2 \mathrm{O}$ have the same warming effect as $21 \mathrm{t}$ and $310 \mathrm{t} \mathrm{CO} 2$ respectively, with conversion factors of 21 and 310 respectively, as follows:

$$
C_{\text {total }}=C 1+C 2+C 3=21 \times(C 1+C 2)+C 3 \times 310
$$

In equation (4). Ctotal Total annual carbon emissions from livestock farming in each state and city (million $\mathrm{t}$ ), and $\mathrm{C} 1$ total annual emissions of $\mathrm{CH} 4$ from livestock enteric fermentation in each state and city (million $\mathrm{t}$ ), and C2 total annual emissions of $\mathrm{CH} 4$ from livestock manure management in each state and city (million $\mathrm{t}$ ), the $C 3$ Total annual emissions of $\mathrm{H} 2 \mathrm{O}$ from livestock manure management in each state and municipality (million $\mathrm{t}$ ).

\section{Data source}

The livestock and poultry slaughter and stock numbers used in this article were obtained from the Yunnan Provincial Statistical Yearbook, and the carbon emission factors were obtained from the Guide to Provincial Greenhouse Gas Inventory Preparation. Due to the limitation of data availability, the article selected pigs, cattle, sheep and poultry as representatives of livestock and poultry species in each state and city of Yunnan Province, and the time interval was 2008-2018. The base period for the decoupling coefficient is 2008 , and the study time interval is $2009-2018$.

\section{Results and conclusion}

\subsection{Time-series characteristics of total carbon emissions from the livestock sector}

According to equations (2), (3), (4) and (5) to measure out the carbon emissions of the livestock industry in each state and city of Yunnan Province, the results show that the total carbon emissions of the livestock industry in Yunnan Province decreased from 25,643,900 t in 2008 to $24,758,200 \mathrm{t}$ in 2018 , with an average annual carbon emission of 30,534,500 $\mathrm{t}$ and an average annual growth rate of $0.35 \%$. In terms of the structure of greenhouse gas emissions, the proportion of carbon dioxide equivalent converted from $\mathrm{CH} 4$ emissions increased from $81.15 \%$ in 2008 to $81.16 \%$ in 2018 , and the proportion of carbon dioxide equivalent converted from $\mathrm{N} 2 \mathrm{O}$ emissions decreased from $18.85 \%$ to $18.84 \%$ in 2008 . This indicates that the structure of livestock farming in Yunnan Province has not changed significantly in the last 10 years, with ruminant cattle and sheep farming accounting for a relatively large proportion, resulting in $\mathrm{CH} 4$ emissions from enteric fermentation becoming the main source of greenhouse gases.

On the whole, the total carbon emissions from the livestock industry in Yunnan Province can be divided into two stages: "rising and falling". In the first stage, from 2008 to 2014, the total carbon emissions from livestock farming rose from $25,739,400$ tons to $33,868,400$ tons, with an average annual carbon emission of 29,411,000 tons and an average annual growth rate of $4.68 \%$. In terms of farming structure, the proportion of carbon emissions from pig farming increased from $12.68 \%$ to $16.85 \%$, while the proportion of carbon emissions from cattle farming decreased from $78.33 \%$ to $74.42 \%$, remaining the main source of carbon emissions from livestock farming. The share of carbon emissions from sheep farming increased from $8.84 \%$ to $9.52 \%$. This shows that during this period, influenced by the government's policy of 
encouraging the development of livestock farming, the number of livestock farming is increasing year by year, but the level of development of scale and intensification is low, and the total carbon emissions have increased, bringing great pressure to the environment.

In the second phase, from 2015 to 2018 , the total carbon emissions from livestock decreased from $34,745,800 \mathrm{t}$ to $24,758,200 \mathrm{t}$, with an average annual carbon emission of $32,783,500 \mathrm{t}$ and an average annual growth rate of $-6.07 \%$. At the same time the proportion of carbon emissions from livestock manure management decreased from $31.57 \%$ in 2015 to $29.96 \%$ in 2018 during the period, and enteric fermentation carbon emissions are still the main source of carbon emissions from the livestock industry. Analysis of the data reveals that since the implementation of the "strictest environmental protection law" in 2015, the prevention and control of pollution from large-scale livestock and poultry farming has become the focus of treatment, and the growth rate of total large- scale livestock and poultry farming has slowed down, making the growth rate of total carbon emissions in this period significantly lower than before. At the same time, the Yunnan provincial government has introduced policies on the resource utilization of livestock and poultry farming waste, and the management of manure in livestock and poultry farming has become more diversified and low-carbon, resulting in a significant decrease in the proportion of carbon emissions from manure management.

\subsection{Analysis of the spatial characteristics of carbon emissions from the livestock industry}

In order to establish a unified standard and horizontal comparability of carbon emissions from animal husbandry in different states and municipalities at different times, with reference to the classification methods of Yao Bo et al and Wang Qiang et al, the province was divided into low animal husbandry carbon emission zones $(<0.5)$, medium livestock carbon emission area ( $0.5 \sim 1)$, high livestock carbon emission zone ( $1 \sim 1.5$ ), and high livestock carbon emission area ( $>$ 1.5 In this paper, we study the spatial changes of carbon emissions in each state and city in Yunnan Province from 2000 to 2018.

During the period 2008-2018 Qujing and Wenshan Prefecture were both high livestock carbon emission zones, while Xishuangbanna, Dehong, Nujiang and Diqing were all low livestock carbon emission zones. Dali Prefecture changed from a high livestock carbon emission area to a partial high livestock carbon emission area, Honghe Prefecture changed from a partial high livestock carbon emission area to a high livestock carbon emission area, and Yuxi changed from a medium livestock carbon emission area to a low livestock carbon emission area. Qujing, Wenshan, Dali and Honghe are the main grainproducing areas in Yunnan Province, and most of the livestock industry layout plans rely on the main grainproducing areas to reduce the production costs of upstream feed processing enterprises, resulting in the concentration of livestock industries in the province. In recent years, the industrial structure of Dali Prefecture has been adjusted to develop the tertiary industry, mainly tourism, which has had a certain impact on the livestock industry and has led to a decline in total carbon emissions. The grain sowing area and grain production in Honghe Prefecture have increased year by year, making the livestock breeding carrying capacity and carbon emissions grow in tandem. Among the low livestock carbon emission zones, Nujiang and Diqing Provinces are located in the northwest of Yunnan Province, which is the highest altitude in Yunnan Province and the terrain is mainly mountainous, which is not conducive to livestock development. Xishuangbanna and Dehong Provinces, due to their unique geographical environment and climate, have been developing mainly in the tertiary sector, with the primary sector accounting for a small proportion and the lowest carbon emissions from animal husbandry in the province.

Yuxi has gradually become an important vegetable growing region in Yunnan Province in recent years, becoming an important vegetable base for Hong Kong and Macau. The area under vegetable cultivation has been increasing year by year and the proportion of livestock farming has been decreasing, making it a low livestock carbon emission area year by year.

Between 2008 and 2018, the number of high livestock carbon emission areas in Yunnan Province decreased from five to three, and the distribution developed from a "circular" to a "linear" one around Yuxi. The number of medium livestock emission zones did not fluctuate much, but their share of total emissions increased from 19.54\% to $27.8 \%$ in that year. Kunming, Pu'er and Lincang show an evolution from high to medium livestock carbon emission zones, and according to the analysis of the data, it can be predicted that the carbon emissions from livestock in these three areas will still show a decreasing trend in the next few years, and are expected to develop into low livestock carbon emission zones. Both Baoshan and Honghezhou show an upgrading of the livestock carbon emission classification area, with Baoshan changing from a medium to a high livestock carbon emission area and Honghezhou changing from a high to a high livestock carbon emission area. This indicates that the development of the livestock industry in Yunnan Province is gradually shifting towards the main grainproducing areas, with each state and municipality making dynamic adjustments to the development of the livestock industry according to the actual situation of their own agricultural economic development. It is not conducive to the stable development of animal husbandry.

\section{References}

1. Steinfeld H, Gerber P, Wassenaar T, et al. Livestock's Long Shadow: Environmental Issues and Options[R]. Rome: FAO, 2006.

2. Yao, Chengsheng, Qian, Shuangshang, Li, Zhengtong, Liang, Longwu. Carbon emission measurement and spatial and temporal evolution mechanism of inter-provincial animal husbandry in China[J]. Resource Science, 2017, 39(04): 698-712. 
3. Chen Yao. Study on the measurement of carbon emission and the path of sink enhancement and emission reduction in China's livestock industry[D]. Northeast Forestry University,2016.

4. Xie Ting, Zhang Hui, He Jiajun, Liu Yu, Huang Kaiwei, Song Mingwei. Characterization and prediction of greenhouse gas emissions from animal husbandry in central China[J]. China Environmental Science,2020,40(02):564-572.

5. Chen Su, Hu Hao. Study on the spatial and temporal changes of greenhouse gas emissions from livestock and poultry in China and the influencing factors[J]. China Population-Resources and Environment, 2016, 26(07): 93-100. 